\title{
A radiographic analysis of tooth morphology following the use of a novel cyclical force device in orthodontics
}

\author{
Chung H Kau
}

\begin{abstract}
Background: The purpose was to determine whether or not a novel device used in conjunction with orthodontic treatment produced root resorption shown on 3D images generated from a new cone beam computerized tomography.

Methods: Subjects were actively recruited and those who received braces for the first time were invited to participate. Patients were assigned to receive a functioning device and used the devices for 20 min daily for a six month study period. CBCT images were taken of the dentition at the start of treatment and at the end of the study period.
\end{abstract}

Results: 14 subjects out of a possible 17 subjects completed using the device during the study period. The mean age of the subjects was 20.3 years. Measurements of all teeth present were made from the mesial buccal roots of the first molar on one side of the dental arch to the mesial buccal roots of the first molar on the opposing side of the same arch. These measurements were recorded as linear lengths in $\mathrm{mm}$. A paired $t$-test was used to determine if significant differences occurred for root lengths at the end of treatment compared to the start of treatment for each of the individual tooth groups. No statistical differences were noted for root length changes above $0.5 \mathrm{~mm}$ and $1 \mathrm{~mm}$.

Conclusions: No statistically significant findings were noted for root length change at the end of treatment compared to the start of treatment when using this novel robotic device. No significant differences were noted between roots of anterior and posterior teeth. No clinically significant changes between root lengths were noted above $0.5 \mathrm{~mm}$.

\section{Introduction}

The clinical practice of orthodontics has been based on movement of teeth through alveolar bone using biomechanical methods within a safe, cellular environment. This technique involves the use of static mechanical forces to move teeth within the jawbone. The most common treatment approach is to correct malocclusion by providing these mechanical forces. This treatment has been used for approximately 100 years and involves a system of metal archwires and brackets, typically referred to as orthodontics. The basic system may be augmented with elastics, metal bands, head gear,

Correspondence: ckau@uab.edu

Department of Orthodontics, University of Alabama at Birmingham School of Dentistry, 1919 7th Avenue South, Room 305, Birmingham, AL 35294, USA retainers, and other ancillary devices as dictated by the specific and individualized treatment. These forces are static in that they are only adjusted at specific visits but then stay constant and do not change between visits.

Orthodontics works by applying steady pressure to the teeth (static forces), moving them gently and gradually into new positions according to the interaction of the archwire and bracket. Physiologically, this is possible because bone is constantly remodelling. When a tooth is pushed in a certain direction, the surrounding bone is remodelled. The direction of bending of the tooth is influenced by polarity created by the mechanical forces. When the tooth is under pressure and increased in convexity, the area is in an electropositive state. This state is associated with osteoclastic activity of bone resorption. When the tooth is under tension and increased in
C Biomed Central

(c) 2011 Kau; licensee BioMed Central Ltd. This is an Open Access article distributed under the terms of the Creative Commons Attribution License (http://creativecommons.org/licenses/by/2.0), which permits unrestricted use, distribution, and reproduction in any medium, provided the original work is properly cited. 
concavity, the area is in an electronegative state. This state is associated with osteoblastic activity of bone deposition [1].

Tooth movement may be considered an inflammatory process, and cytokines, such as interleukin-1 (IL-1), interleukin-6 (IL-6), and receptor activator of nuclear factor $\kappa \mathrm{B}$ ligand (RANKL), are inflammatory or pro-inflammatory mediators remodelling the periodontal ligament (PDL) tissue [2]. The PDL is a connective tissue attaching the tooth to the alveolar bone. The tissue withstands the compressive forces during chewing while keeping the tooth in place. RANKL is reportedly essential to the osteoclast formation, function, and survival [3].

Some orthodontic researchers have suggested other methods to increase the rate of tooth movement by exploiting cellular processes. One such method is the use of corticotomies to accelerate tooth movement [4]. A recent article has even suggested that different types of surgical procedures create different effects in the surrounding bony areas facilitating a variable response to tooth movement [5].

In another study, it has been reported that low magnitude mechanical signals are "anabolic" to bone when applied at a high frequency. Long term use of this technique enhances bone stiffness and strength, and it also shows an increase in cancellous bone volume fraction, trabecular thickness, and trabecular number [6]. A light force produces significantly more tooth movement than heavier force application [7]. However, optimal force varies between patients along with the magnitude of the applied force affecting the rate of tooth movement [2]. Therefore, a device that transmits these forces may be an added benefit in orthodontic treatment.

However, use of such a device may pose a potential problem in root resorption. This condition is characterized by the loss of root cementum and dentin [8]. As a result, root resorption is a concern in orthodontic treatment and is thought to occur as a side-effect of cellular activity in the removal of the necrotic hyalinized tissue [2]. Root resorption is a precursor to the eruption of permanent teeth. However, root resorption of permanent teeth is an inflammation caused by varying factors, including injury to the root surface followed by dental trauma, surgical procedures, non-vital teeth bleaching, and mechanical procedures involving periodontal treatment [8].

The "gold standard" to measure root resorption is to sacrifice the tooth and surrounding alveolar bone and to histologically analyze the morphology. However, this type of analysis is not possible in a clinical setting. Therefore, a common method of evaluating root resorption is through conventional radiography. Some examples are panoramic radiography or peri-apical films. However, these models may be of limited use. A more accurate evaluation of root resorption can be achieved by analyzing cone beam computed tomography (CBCT) images. CBCT imaging has been moving toward providing greater amounts of information in regard to root morphology and periodontal structures [9].

This study represents the first human use of a novel cyclical device. The purpose of this study was to determine the effects a cyclical device may have on root lengths of teeth on 3D images generated from a new, computerized cone beam tomography device.

\section{Methods}

Subjects who received braces for the first time were invited to participate, as long as they were within the first week of getting braces bonded. Patients were assigned to receive a functioning device and used the devices for 20 min daily for a six month study period. Study approval was given by the Institutional Review Board (IRB) at the University of Texas Health Science Center, Houston, TX, USA.

The inclusion criteria for subjects were as follows:

\section{Permanent dentition}

2. Class I malocclusion with crowding or spacing of $\geq 6 \mathrm{~mm}$ for mandibular incisors, lower number 1's through 3's

3. All patients will be candidates for canine retraction with bicuspid extraction

4. Predicted compliance with device use, as determined by the investigator orthodontist

5. Good oral hygiene, as determined by the investigator orthodontist

6. At least average intelligence, as determined by investigator orthodontist

The exclusion criteria for subjects were as follows:

1. Any medical or dental condition that in the opinion of the investigator could impact study results during the expected length of the study

2. Patient is currently using any investigational drug or any other investigational device

3. Patient plans to relocate or move within six months of enrollment

4. Allergic to acetaminophen (use of aspirin or nonsteroidal anti-inflammatory drugs is excluded for patients while on the study)

5. Use of bisphosphonates, such as osteoporosis drugs, during the study

6. Pregnancy

\section{Novel device}

The novel device used for this study was the AcceleDent Type I (Figure 1). The device uses the application 


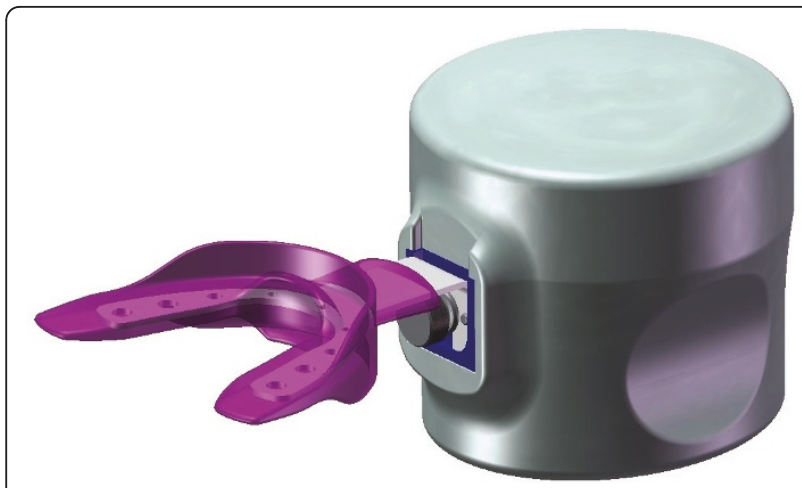

Figure 1 An example of the AcceleDent Type 1 device.

of cyclic forces to move teeth in bone faster through accelerated bone remodelling. The product is a removable orthodontic device, similar to a retainer, which attaches to the orthodontic archwire. In short, one part of the device is placed into the subject's mouth while the other end sits just outside the mouth and provides a small mechanical force to the teeth. The component outside the mouth shaped like a computer mouse and houses the mechanical, electrical, and energy components to activate the mechanical force from the post. The patient places and activates the device once daily for $20 \mathrm{~min}$. The applied force (0.2-10 Newtons) is intended to be barely noticeable and should not be uncomfortable. Some researchers have theorized that the pulsing actually may decrease pain associated with standard orthodontic adjustments [10]. Importantly, AcceleDent is designed to work with all existing bracket technologies and is intended to complement rather than replace existing bracket technologies, such as braces.

\section{Imaging Device}

The CBCT imaging device used for this study was the Sirona Galileos cone beam device. This system emits a radiation dose between $29 \mathrm{uSv}$ to $54 \mathrm{uSv}$, as reported by the manufacturer. It has a scan time of $14 \mathrm{~s}$ and captures the maxilla-mandibular region in a $210^{\circ}$ rotation within a radiation-detector configuration. The field of view is a spherical volume of $15 \mathrm{~cm}$. The voxel size is between $0.15 \mathrm{~mm}$ to $0.30 \mathrm{~mm}$, and the grayscale is 12 bit.

A reconstruction program calculated the entire image volume from the data of 200 individual exposures generated from a pulsed scan and required $3 \mathrm{~min}$ for image generation. Image manipulation was carried out using the manufacturer's software, Galaxis. To increase the accuracy of the assessment, all three planes (sagittal, axial, and coronal) were utilized.

\section{Parameters Measured}

CBCT images were taken at two time frames; once at the start of treatment $\left(\mathrm{T}_{1}\right)$ and again after six months of treatment $\left(\mathrm{T}_{2}\right)$. Measurements of all teeth present were made from the mesial buccal roots of the first molar on one side of the dental arch to the mesial buccal roots of the first molar on the opposing side of the same arch (Figure 2). Linear root measurements were recorded in mm.

A further analysis was done to determine if groups of teeth reacted differently. For example, if the anterior teeth (canines and incisors) reacted differently to the posterior teeth (premolars and molars).

\section{Statistical Analysis}

The mean of the root lengths were measured in $\mathrm{mm}$ and tested for normality. The differences between the pre-treatment and mid-treatment root lengths were analyzed by using $t$-tests (SPSS 16.0.1, Chicago, IL). Reductions in tooth root length were measured for significant differences at $0.5 \mathrm{~mm}$ and $1 \mathrm{~mm}$.

\section{Results}

The following results were obtained, and some of the results are presented in Tables 1 and 2 .

\section{Subjects}

17 subjects were recruited to participate in the study. 14 subjects completed using the device during the study period. 3 subjects declined to continue using the device for a variety of personal reasons and were not included in this study. The mean age of the subjects was 20.3 years. The oldest patient was 56.6 years, and the youngest was 12.1 years.

\section{Mean Root Lengths}

Measurements of all teeth present were made from the mesial buccal roots of the first molar on one side of the

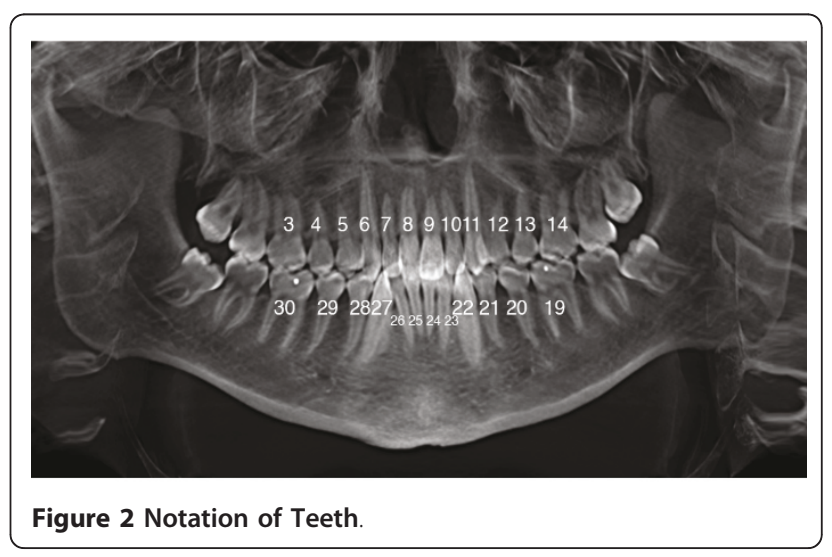


Table 1 Table showing the mean changes in root lengths at $T_{2}$ compared to $T_{1}$

\begin{tabular}{|c|c|c|c|c|c|c|c|}
\hline Teeth & $\mathrm{N}$ & Mean $(\mathrm{mm})$ & Std Dev $(\mathrm{mm})$ & $\operatorname{Max}(\mathrm{mm})$ & $\operatorname{Min}(\mathrm{mm})$ & P Sig at $0.5 \mathrm{~mm}$ & P Sig at $1 \mathrm{~mm}$ \\
\hline 3 & 14 & -0.127 & 0.226 & 0.4 & -0.58 & NS & NS \\
\hline 4 & 14 & -0.034 & 0.457 & 1.19 & -0.75 & NS & NS \\
\hline 5 & 11 & -0.103 & 0.449 & 0.75 & -0.85 & NS & NS \\
\hline 6 & 14 & -0.416 & 0.316 & 0.01 & -0.92 & NS & NS \\
\hline 7 & 14 & -0.112 & 0.295 & 0.39 & -0.66 & NS & NS \\
\hline 8 & 14 & -0.12 & 0.322 & 0.37 & -0.746 & NS & NS \\
\hline 9 & 14 & -0.321 & 0.341 & 0.19 & -1.07 & NS & NS \\
\hline 10 & 14 & -0.295 & 1.005 & 1.28 & -3.39 & NS & NS \\
\hline 11 & 14 & 0.176 & 1.453 & 5.1 & -1.06 & NS & NS \\
\hline 12 & 11 & -0.222 & 0.234 & 0.19 & -0.58 & NS & NS \\
\hline 13 & 14 & 0.173 & 0.766 & 2.62 & -0.47 & NS & NS \\
\hline 14 & 14 & -0.047 & 0.409 & 1.08 & -0.57 & NS & NS \\
\hline 19 & 13 & -0.107 & 0.205 & 0.13 & -0.5 & NS & NS \\
\hline 20 & 12 & 0.271 & 0.804 & 2.54 & -0.56 & NS & NS \\
\hline 21 & 14 & -0.176 & 0.562 & 1.06 & -1.12 & NS & NS \\
\hline 22 & 14 & -0.06 & 0.48 & 1.14 & -0.67 & NS & NS \\
\hline 23 & 14 & -0.081 & 0.163 & 0.29 & -0.44 & NS & NS \\
\hline 24 & 14 & -0.284 & 0.44 & 0.29 & -1.38 & NS & NS \\
\hline 25 & 14 & -0.336 & 0.442 & 0.18 & -1.27 & NS & NS \\
\hline 26 & 14 & -0.302 & 0.613 & 0.64 & -1.83 & NS & NS \\
\hline 27 & 14 & -0.079 & 0.686 & 2.12 & -0.72 & NS & NS \\
\hline 28 & 14 & 0.076 & 1.047 & 3.45 & -0.69 & NS & NS \\
\hline 29 & 13 & -0.225 & 0.383 & 0.24 & -1.27 & NS & NS \\
\hline 30 & 13 & -0.142 & 0.351 & 0.28 & -0.74 & NS & NS \\
\hline
\end{tabular}

The $p$ values at $0.5 \mathrm{~mm}$ and $1 \mathrm{~mm}$.

dental arch to the mesial buccal roots of the first molar on the opposing side of the same arch. Measurements were recorded as linear lengths. The mean root lengths of the upper and lower teeth are presented in Table 1. The differences in mean root lengths ranged from $-0.127 \mathrm{~mm}$ to $-0.416 \mathrm{~mm}$ for both arches.

\section{Parameters measured}

A paired $t$-test was used to determine if significant differences in root lengths occurred at the end of the study period compared to the start of treatment for each of the individual tooth groups. No statistical differences were noted for root length changes above $0.5 \mathrm{~mm}$ and $1 \mathrm{~mm}$.

When groups of teeth were measured, the results showed no statistical differences in the amounts of root resorption between anterior and posterior teeth (Table 2).

\section{Discussion}

This was the first study conducted in humans to determine the safety and efficacy of a novel device that uses medical robotics to assist in the rapid movement of teeth. State of the art 3D technology was employed to determine if the device caused problems to the roots of all teeth and whether root resorption occurred.

The device used in this study was the AcceleDent Type 1 device. This device provides a cyclical force in addition to the standard static force provided by orthodontics. Application of these cyclical forces induces accelerated remodelling of the bone in which teeth are embedded, thereby enabling them to move faster. In a series of rabbit experiments $(\mathrm{N}=24)$, Mao showed that cyclical forces $(2$ Newtons at $0.2 \mathrm{~Hz}$ and $1 \mathrm{~Hz}$ for 20 min daily), provided in addition to the typical static

Table 2 Means of the differences in root lengths at $T_{2}$ compared to $T_{1}$ based on groupings of anterior and posterior teeth

\begin{tabular}{cccc} 
Group & Mean & Std Dev & $\begin{array}{c}\text { Std Err } \\
(<\mathbf{0 . 0 5})\end{array}$ \\
\hline Anterior Teeth (Maxilla vs Mandible) & -0.01 & 0.65 & 0.10 \\
Anterior Teeth versus Posterior Teeth(maxillia) & 0.13 & 0.64 & 0.10 \\
Anterior Teeth verus Posterior Teeth (mandible) & -0.14 & 0.57 & 0.09 \\
\hline
\end{tabular}

T-test indicated no statistically significant differences in groupings. 
forces (braces provided 24 hours per day), induced more cranial growth, sutural separation, and proliferation of osteoblast-like cells $[11,12]$. Histological evidence indicated wider separation of the premaxillomaxillary suture, frontonasal suture, and maxillopalatine suture associated with cyclic loading. In contrast, sutures associated with control and static loads were less separated. This evidence provides the scientific basis for using a cyclical device to decrease standard orthodontic treatment time. Additionally, a device that utilizes cyclic forces has been applied and approved for use in other areas of the body [13]. For example, the Juvent 1000 device maintains and/or enhances muscle strength, function, and postural stability.

Root resorption is a potential side effect of any orthodontic treatment. However, numerous factors have been acknowledged as potential precursors to enhanced root resorption. These factors include the duration of treatment, the magnitude of force application, the direction of tooth movement, and the method of force application (continuous versus intermittent) [8].

In this study, the AcceleDent device was used as an adjunct to routine treatment. The types of forces were cyclical in nature hence providing an almost pulsating nature. In addition, the device was used for only $20 \mathrm{~min}$ a day. The closest force characteristic that this device produced would be seen as an intermittent force, and these types of forces have been shown to allow cementum to heal and prevent further resorption [14-16].

Furthermore, there have been conflicting discussions of what is considered to be clinically significant root resorption. Some authors have stated that root resorptions in excess of $1 / 3$ of root length were significant [17] whilst another study showed that resorptions at $>2$ $\mathrm{mm}$ were considered present in up to $25 \%$ of cases [18]. This study showed that the changes in the root lengths at the end of the treatment compared to the start of treatment were not statistically significant at the $0.5 \mathrm{~mm}$ and $1 \mathrm{~mm}$ levels. This stringent amount of $0.5 \mathrm{~mm}$ was considered to be within clinically acceptable limits considering the study lasted for 6 months, and long term results were not available.

\section{Conclusions}

The following are conclusions of the novel robotic device. No statistically significant changes were noted for root lengths at the end of treatment compared to the start of treatment. No significant differences were noted between roots of anterior and posterior teeth. No clinically significant changes between root lengths were noted above $0.5 \mathrm{~mm}$.

\section{Competing interests}

The author declares that they have no competing interests.

Received: 18 April 2011 Accepted: 9 August 2011

Published: 9 August 2011

\section{References}

1. Darendeliler MA, Zea A, Shen G, Zoellner H: Effects of pulsed electromagnetic field vibration on tooth movement induced by magnetic and mechanical forces: a preliminary study. Aust Dent J 2007, 52:282-287.

2. Meikle MC: The tissue, cellular, and molecular regulation of orthodontic tooth movement: 100 years after Carl Sandstedt. Eur J Orthod 2006, 28:221-240.

3. Nishimura M, Chiba M, Ohashi T, Sato M, Shimizu Y, Igarashi K, et al: Periodontal tissue activation by vibration: intermittent stimulation by resonance vibration accelerates experimental tooth movement in rats. Am J Orthod Dentofacial Orthop 2008, 133:572-583.

4. Wilcko WM, Wilcko T, Bouquot JE, Ferguson DJ: Rapid orthodontics with alveolar reshaping: two case reports of decrowding. Int J Periodontics Restorative Dent 2001, 21:9-19.

5. Lee W, Karapetyan G, Moats R, Yamashita DD, Moon HB, Ferguson DJ, et al: Corticotomy-/osteotomy-assisted tooth movement microCTs differ. J Dent Res 2008, 87:861-867.

6. Rubin C, Judex S, Qin YX: Low-level mechanical signals and their potential as a non-pharmacological intervention for osteoporosis. Age Ageing 2006, 35(Suppl 2):ii32-ii6.

7. Gonzales C, Hotokezaka H, Yoshimatsu M, Yozgatian JH, Darendeliler MA, Yoshida N: Force magnitude and duration effects on amount of tooth movement and root resorption in the rat molar. Angle Orthod 2008, 78:502-509.

8. Pizzo G, Licata ME, Guiglia R, Giuliana G: Root resorption and orthodontic treatment. Review of the literature. Minerva Stomatol 2007, 56:31-44.

9. Kau CH, Richmond S, Palomo JM, Hans MG: Three-dimensional cone beam computerized tomography in orthodontics. J Orthod 2005, 32:282-293.

10. Ste Mare S, Powers M, Sheridan J: Vibratory Stimulation as a Method of Reducing Pain after Orthodontic Appliance Adjustment..

11. Mao JJ: Mechanobiology of craniofacial sutures. J Dent Res 2002, 81:810-816.

12. Mao JJ, Nah HD: Growth and development: hereditary and mechanical modulations. Am J Orthod Dentofacial Orthop 2004, 125:676-689.

13. Eisman JA: Good, good, good... good vibrations: the best option for better bones? Lancet 2001, 358:1924-1925.

14. Faltin RM, Faltin $K$, Sander FG, Arana-Chavez VE: Ultrastructure of cementum and periodontal ligament after continuous intrusion in humans: a transmission electron microscopy study. Eur J Orthod 2001, 23:35-49.

15. Acar A, Canyurek U, Kocaaga M, Erverdi N: Continuous vs. discontinuous force application and root resorption. Angle Orthod 1999, 69:159-163.

16. Konoo T, Kim YJ, Gu GM, King GJ: Intermittent force in orthodontic tooth movement. J Dent Res 2001, 80:457-460.

17. Lupi JE, Handelman CS, Sadowsky C: Prevalence and severity of apical root resorption and alveolar bone loss in orthodontically treated adults. Am J Orthod Dentofacial Orthop 1996, 109:28-37.

18. Sameshima GT, Sinclair PM: Predicting and preventing root resorption: Part II. Treatment factors. Am J Orthod Dentofacial Orthop 2001, 119:511-515.

doi:10.1186/1746-160X-7-14

Cite this article as: Kau: A radiographic analysis of tooth morphology following the use of a novel cyclical force device in orthodontics. Head \& Face Medicine 2011 7:14. 